\title{
Clinical Evaluation and Investigations in Women with Stress Urinary Incontinence: An Overview
}

\author{
Arshiya SULTANA ${ }^{1}$, Padmaja A. RANGASWAMY ${ }^{2}$, Khaleeq ur RAHMAN ${ }^{3}$, Tripura SUNDARI ${ }^{4}$
}

${ }^{1}$ Department of Amraze Niswan wa IImul Qabalat (Gynecology and Obstetrics), National Institute of Unani Medicine, Bangalore, Karnataka, India.

${ }^{2}$ Kegalle Ayurvedic Hospital, Srilanka.

${ }^{3}$ Department of Ilmul Saidla (Pharmacy), National Institute of Unani Medicine, Bangalore-Karnataka, India.

${ }^{4}$ Gandhi Medical College, Hyderabad-AP, India.

\section{ABSTRACT}

Urinary incontinence (UI) is defined as the complaint of any involuntary loss of urine. UI can be slightly bothersome or totally debilitating. For some women, the risk of public embarrassment prevents them from enjoying many activities with their family and friends. Women are four times more likely to be affected by UI compared with men. Stress urinary incontinence (SUI) is the most common type of UI in women below age 60 and accounts for almost $50 \%$ of incontinence in all women. The minimum evaluation in women with symptoms of uncomplicated SUI includes history, urinalysis, physical examination, assessment of urethral mobility, demonstration of SUI, and measurement of postvoid residual urine volume. Additional diagnostic evaluation with multichannel urodynamic testing is helpful in women with complicated SUI, especially before surgical treatment. Clinical decision should help the health care providers to perform preoperative multichannel urodynamic testing or refer to an expert in urology who is skilled in female pelvic medicine and reconstructive surgery.

Key words: Clinical evaluation, diagnosis, stress urinary incontinence, urinary incontinence, urodynamic studies

\section{INTRODUCTION}

Urinary incontinence (UI) is one of the manifestations of pelvic floor dysfunction and defined as the complaint of any involuntary loss of urine that can be objectively demonstrated $(1,2)$. On the contrary, continence is the ability to hold urine within the bladder at all times except during micturition (3). The continence mechanism includes a sophisticatedly organized series of nerves, muscles, and connective tissue that dynamically influence bladder control. This arrangement allows prompt and complete bladder emptying and also maintains continence during tremendous increases in abdominal pressure (4). Both continence and micturition depend upon the lower urinary tract, consisting of the bladder and urethra, which is structurally and functionally normal (3). Women are four times more likely to be affected by UI compared with men (5). Thomas et al. have shown that Ul occurs twice or more per month in at least a third of the female population above the age of 35 years (3).

Female $\mathrm{UI}$ is a public health problem due to its high prevalence (200 million people around the world), significantly poor quality of life and depression, and cost of its management (6). The Clinical Practice Guideline issued by the Agency for Health Care Policy and Research defined four types of UI: stress, urge, mixed, and overflow. Some authors include functional incontinence as a fifth type of incontinence (7). SUI, urge urinary incontinence (UUI), and mixed urinary incontinence (MUI) are the three most common types of $U$ I (8). The most common type of $U$ I is stress urinary incontinence (SUI) (9). The International Consultation on Incontinence defines SUI as an involuntary loss of urine on physical exertion, sneezing or coughing (10), and a rise in abdominal pressure (11-14). 
The prevalence of $U I$ is expected to increase with a change in demographics and an increase in the elderly population (10). Female patients with UI are increasingly seen in the physician's office because of its high prevalence (20\%-30\% of middle-aged and $30 \%-50 \%$ of elderly women) and growing expectations for relief by women affected by it $(5,16)$.It has been estimated that $21 \%$ of the population will be 65 years and older by the year 2050. Hence, the probable growth in the incontinent patient population presents an alarming challenge to the health care community (5). SUI is a significant clinical problem at any given time with estimates of between $12 \%$ and $44 \%$ of women (17). In general, SUI affects around 50\% incontinent women and a majority of women are young or middle aged. (18). SUI affects up to $24 \%$ of postpartum women (19).

The risk factors for SUI in women include advanced age, advanced pelvic organ prolapse (POP), pregnancy, obesity, constipation, weak collagen, and chronic obstructive airway disease (11). Pathophysiologically, SUI can be the result of bladder neck/ urethral hypermobility and/or neuromuscular defects (intrinsic sphincter deficiency) $(11,13,14)$. Internal sphincter deficiency (ISD) is used to describe damage to the urethral sphincteric mechanism, regardless of etiology. The urethra might be damaged owing to fixation (as in cases of spina bifida), prior surgery, or denervation or muscle damage during childbirth. ISD and hypermobility can exist concomitantly and alone (20).

\section{Diagnosis}

The clinical evaluation of women with $\mathrm{UI}$ includes patient history, physical examination, and measures of incontinence severity (21). Moreover, the clinical evaluation in women with symptoms of uncomplicated SUI includes history, urine examination, physical assessment, revelation of SUI, evaluation of urethral mobility, and postvoid residual (PVR) urine volume measurement (22).

- History: History provides an important basis for treatment, although it is not diagnostic (23). The reason for history taking is to find out the type of $\mathrm{UI}$ that is troublesome to the patient, which is commonly classified as stress, urge, postural, insensible (spontaneous), continuous, coital, overflow (chronic urinary retention), nocturnal enuresis, or some combination thereof (22). Additionally, while defining symptoms related to the type of incontinence, it is important to know the duration of symptoms, previous treatment for this condition and outcome of treatment, previous surgery for this condition or other related conditions, and activities that produce or exacerbate symptoms (5). The number of voids, type of pad, frequency of pad used and changed per day, and the amount of pad saturation are major considerations (24). To assess symptoms related to bladder storage (frequency, nocturia, urgency, and incontinence) and emptying functions (hesitancy, intermittency, slow stream, feeling of incomplete emptying, straining to void, spraying of urinary stream, postmicturition leakage, need to immediately revoid, position-dependent micturition, and dysuria), the patients are interrogated (1). UI is a major quality-of-life issue, and it is essential to assess to what degree the patient's symptoms impact her lifestyle $(1,3)$. Several disease-specific quality-of-life instruments have been developed to provide detailed information on how UI affects patient lives (25). Validated questionnaires such as Incontinence Severity Index, Questionnaire for Urinary Incontinence Diagnosis, and Urogenital Distress Inventory are useful for assessing the quality of life. Incontinence Impact Questionnaire, Incontinence-Quality of Life Questionnaire, International Consultation on Incontinence Questionnaire $(3,22)$, and King's Health Questionnaire (3) are useful for evaluating the severity and relative contribution of UUI and SUI symptoms. Women with uncomplicated SUI have characteristic symptoms of leakage on effort or physical exertion. A functional component to the incontinence is typically ruled out in absence of cognitive impairment, and fistula is ruled out in absence of continuous leakage in women with recent pelvic surgery or radiation exposure points. Following the urologic history, systematic medical and neurologic histories are elicited (22).

- Past medical history: In addition to specific details describing urinary symptoms, other medical factors may be associated with incontinence. Obstetric trauma may be associated with damage to the pelvic floor support, which may lead to SUI. For this reason, information describing a prolonged labor, operative vaginal 
delivery, macrosomia, postpartum catheterization for urinary retention, and increased parity may be valuable. Prior radiation therapy for malignancy may lead to irritative voiding symptoms or intrinsic sphincteric deficiency, which predisposes to SUI (23). Diabetes, thyroid disease, and neurologic disorders can cause UI (5). Moreover, drug history (including nonprescription medications) should be obtained to find out the influence of any individual drug on the function of the bladder or urethra that causes UI or voiding difficulties (26). Medicines that can affect lower urinary tract function include anticholinergics, analgesics, calciumchannel blockers, antihistamines, psychotropic drugs, caffeine, alcohol, diuretics, narcotic, drugs, alpha-adrenergic blockers, and alpha-adrenergic agonists. Additionally, gynecologic, obstetric, and surgical histories should be obtained. The Third International Consultation on UI recommends outcome measures, such as voiding diaries, pad tests, or quality-of-life questionnaires. However, due to time constraints, logistic difficulties, and lack of familiarity with the tools, the aforementioned measures are seldom used by clinicians in routine practice (27).

- Urinary symptoms: Seventy-seven percent women with SUI report their symptoms to be troublesome, and of these, $28.8 \%$ report their symptoms to be moderately to extremely worrisome; the degree of problem is related to the severity of SUI (28). Most women may void eight times per day or less. Increased voiding may indicate UUI, urinary tract infection (UTI), calculi, or urethral pathology without a history that reflects increased fluid intake. This needs prompt additional evaluation. Furthermore, urinary frequency is commonly associated with interstitial cystitis (IC), and women with IC may void more than 20 times per day. In women with UUI or in those with systemic fluid management disorders such as congestive heart failure, nocturia may be noted $(5,24)$. Diagnostic clues are also provided by the volume of urine lost with each episode. Spontaneous detrus or contractions associated with UUI typically lead to loss of large volumes and may frequently involve loss of the entire bladder volume. On the contrary, women with SUI usually describe loss of smaller volumes and are often able to contract the levator ani muscles to temporarily stop their urine stream. Postvoid dribbling is characteristically associated with urethral diverticulum, which may frequently be mistaken for UI. Hematuria, even though a common sign of UTI, may also show underlying malignancy and can cause irritative voiding symptoms. The onset of symptoms may also give information regarding the etiology and treatment. For example, the onset of symptoms with menopause may suggest that a hypoestrogenic state underlies incontinence. These patients may benefit from estrogen replacement. In contrast, symptoms after hysterectomy or childbirth may reflect changes in tissue support or innervations $(1,5,24)$. Postural $U I$ is related to a change of body position. Nocturnal enuresis occurs during sleep. MUI involves both UUI and SUI. Continuous UI is continuous involuntary loss of urine. In insensible UI, a woman is unaware how it has occurred. UI occurring with coitus is coital incontinence; it can occur with penetration or intromission and at orgasm (29).

- Physical Examination: Abdominal examination includes bladder fullness/retention, abdominal masses or ascites, and scars (indicating previous relevant surgery or traumas). Renal area examination is done for tenderness and masses $(1,30)$.

General inspection and neurologic evaluation: Initially, the perineum is inspected for the evidence of atrophy, which may be noted throughout the lower genital tract. Additionally, suburethral bulging may indicate a urethral diverticulum and should be excluded during inspection $(24,31)$.

A detailed neurologic evaluation of the perineum is also important for a woman with incontinence, as neurologic responses may be altered in an anxious patient who is in a susceptible setting; signs obtained during examination may not signify true pathology and should be interpreted with caution. Absence of bulbocavernosus reflex may reflect central or peripheral neurologic deficits. A normal circumferential anal sphincter contraction, colloquially called an "anal wink," should follow cotton swab brushing of the perianal skin. Thus, an absent wink may point out neurologic deficits in this neurologic distribution $(5,24)$.

The vaginal examination is usually performed with the woman's bladder empty.

Vulval/urethral examination: Abnormalities such as atrophic changes, cysts, lichen sclerosis, or other tumors are inspected on vulval examination. Urethra is examined for urethral mucosal 
prolapse, urethral caruncle, and diverticulum or urethral discharge on massage of urethra. Urethral diverticulum might be mistaken as a lump or tenderness along the line of the urethra $(3,5,29)$.

Bimanual and rectovaginal examination: In general, these portions of the pelvic examination provide fewer diagnostic clues to the underlying causes of incontinence. Vaginal examination is conducted to examine vaginal length and mobility, scarring and/ or pain, and estrogenization. The location of any vaginal pain should be noted (29). A positive cough stress test confirms the diagnosis of SUI $(5,31)$.

However, bimanual examination may reveal an enlarged pelvic mass or a uterus enlarged by leiomyomas or adenomyosis. These may prompt incontinence through increased external pressure transmitted to the bladder. In addition, stool impaction is common in nursing home patients and may lead to bladder urgency and subsequent UI $(24,31)$.

\section{- Pelvic support assessment:}

POP evaluation: Prolapse coexists with SUI in up to 50\% cases. 33 Poor urethral support usually accompanies POP. For example, women with considerable prolapse are frequently unable to completely empty their bladder due to urethral kinking and obstruction. These women habitually must digitally elevate or reduce their prolapse to allow emptying. Therefore, an external evaluation for POP is specified for all women with UI $(5,24)$.

POP examination should be performed with the woman's bladder empty, since an increase in bladder volume limits the degree of descent of the prolapse (32). The degree of prolapse may be worse later in the day than it is earlier in the day. Descent of one or more of the anterior vaginal wall, posterior vaginal wall, uterus (cervix), or apex of the vagina (vaginal vault or cuff scar after hysterectomy) is called POP (29). Baden-Walker classification system is useful in grading POP. In this system, the hymen is preferred as the landmark (at the vaginal orifice) to evaluate genital prolapse. According to half-way system, uterine prolapse is graded as follows: 0, no prolapse; 1, half way to hymen; 2, to the hymen; 3, half way past hymen; and 4, maximum descent (33). POPQ (Pelvic Organ Prolapse Quantification) system is an adaptation of the Baden-Walker half-way system (34). The main limitation of the
POPQ system is a time-consuming procedure, more complex to learn and communicate verbally, than the original Baden-Walker system. Using the POPQ system, the maximal protrusion of two points ( $\mathrm{Aa}$ and $\mathrm{Ba}$ ) is measured in the anterior vaginal wall, two points (Ap and Bp) in the posterior wall, ( at the cervix, and D at the posterior fornix in the middle compartments. All measurements can be done with a ruler in centimeters. The hymen is used as the reference point (0). Measurements inside the hymen are negative, and measurements outside the hymen are positive. The aforementioned six measurements and the length of the perineal body (pb), from the hymen to the anus, and the genital hiatus (gh) from the hymen to the urethral opening are done while the patient is doing maximum Valsalva. The total vaginal length (tvl) is measured without Valsalva (35). The grading of POPQ includes stage 0 to stage IV. Stage 0 is if no prolapse is confirmed and in stage I, the most distal portion of the prolapse is more than $1 \mathrm{~cm}$ above the level of the hymen. In stage $I$, the most distal portion of the prolapse is $1 \mathrm{~cm}$ or less proximal to or distal to the plane of the hymen, and in stage III, the most distal portion of the prolapse is more than $1 \mathrm{~cm}$ below the plane of the hymen. Complete eversion of the total length of the lower genital tract is > stage IV (24).

Pelvic floor muscle function (PFMF): PFMF is elicited on vaginal or rectal examination in which the pelvic floor muscles are palpated with the examining finger to check muscle quality (for example, symmetry and bulk) and whether or not, and to what degree, a woman can volitionally contract her muscles (31).

Different methods are used to assess voluntary pelvic floor muscle contraction and relaxation by visual inspection, digital palpation (circumferentially), ultrasound dynamometry, electromyography, or perineometry. Factors to be evaluated include muscle strength, muscular endurance, voluntary muscle relaxation, duration, coordination, repeatability, and displacement. It is desirable to document findings for each side of the pelvic floor individually, to recognize any unilateral defects and asymmetry (29). The International Continence Society (ICS) report, evaluation of pelvic floor muscle function, includes normal pelvic floor muscles, overactive pelvic floor muscles, underactive pelvic floor muscles, and nonfunctioning pelvic floor muscles, where no action is palpable (36). 
Examination for levator (puborectalis) injury: The presence of major morphological abnormalities of the puborectalis muscle may be assessed by palpating the insertion of puborectalis on the inferior aspect of the os pubis. Palpation of the bony surface of the os because of the absence of the muscle is noted in an "avulsion injury" of the puborectalis muscle, and the muscle is absent 2-3 cm lateral to the urethra (37).

Perineal examination: The perineum should show no downward movement when the patient is asked to cough or do Valsalva maneuver. Ventral movement may occur because of the guarding actions of the pelvic floor muscles (36).

Rectal examination: Observations can include anal sphincter tone and strength, anal sphincter tear, presence or absence of rectocele and, if possible, differentiated from enterocele, perineal body deficiency, presence or absence of fecal impaction, and other rectal lesions such as intussusception, rectovaginal fistula, tumors, hemorrhoids, fissures, and other perianal lesions (anocutaneous fistula) (29).

- Basic evaluation of Uncomplicated and Complicated SUI:

Uncomplicated SUI: In uncomplicated SUI, women have UIassociated physical exertion, coughing or sneezing without voiding symptoms, absence of recurrent UTI, no history of prior surgery for SUI or extensive pelvic surgery, and medical conditions that can affect lower urinary tract function. On physical examination, a vaginal bulge beyond the hymen and urethral abnormalities are absent and urethral mobility is present. PVR urine volume is less than $150 \mathrm{~mL}$, and urinalysis/urine culture is negative for UTI or hematuria $(3,5)$.

Complicated SUI: In complicated SUI, women present with symptoms of urgency, UI related to chronic urinary retention, incomplete emptying, functional impairment, or continuous leakage. Women may present with a history of current UTI, previous extensive or radical pelvic surgery (e.g., radical hysterectomy), anti-incontinence surgery or complex urethral surgery (e.g., urethral diverticulectomy or urethrovaginal fistula repair), presence of neurologic disease, poorly controlled diabetes mellitus, or dementia. Women may present with voiding symptoms such as the need to immediately revoid, slow stream, hesitancy,
TABLE 1: Investigations of female.

\begin{tabular}{l}
\hline General practitioner/outpatient (Level I) \\
\hline Urinalysis \\
Frequency/volume chart \\
Pad test \\
Stress test \\
Postvoid residual urine volume \\
\hline Simple urodynamic tests (Level II) \\
\hline Filling cystometry \\
Voiding cystometry \\
Subtracted cystometry \\
Q-tip test \\
Bonney's test \\
\hline Complex urodynamic tests (Level II) \\
\hline Multichannel cystometry \\
Uroflowmetry \\
Cystometrography \\
Pressure flowmetry \\
Urethral pressure profile flowmetry \\
Stress leak point pressure \\
Urethrocystoscopy \\
Videocystourethroscopy \\
Urethral electrical conductance \\
Ultrasound \\
Intravenous urography \\
Cysteurethrography \\
Ambulatory urodynamic \\
Combines cystometry, and uroflowmetry with radiological \\
screening of bladder with urethra \\
Magnetic resonance imaging: To show multicompartmental \\
prolapse \\
\hline
\end{tabular}

spraying of urinary stream, intermittency, straining to void, feeling of incomplete voiding, postmicturition leakage, dysuria, and position-dependent micturition. Physical examination confirms vaginal bulge or known POPpelvic organ on examination prolapse beyond the hymen, genitourinary fistula, or urethral diverticulum, and absence of urethral mobility. The PVRpost void residual volume is greater more than or equal to $150 \mathrm{ml} \mathrm{mL}$ (22).

- Diagnostic testing: An accurate diagnosis can be reached with appropriate investigations (3). The test has three levels: basic screening test (level I), simple urodynamic tests (level II), and complex urodynamic tests (level III) (Table 1).

1. Basic screening test (Level I): Urinalysis, voiding dairy, PVR urine measurement (23), stress test, and pad test $(3,38)$. 
- Urinalysis and culture: In all women with UI, urinalysis helps to exclude bacteriuria, hematuria, pyuria, glycosuria, and proteinuria. Urinalysis and urine culture are performed at an initial visit (38). Infection is treated, and persistent symptoms should prompt additional evaluation $(1,23,24)$.

- Frequency-volume chart/Bladder diary: The simple noninvasive assessment such as bladder chart or bladder diary can be extremely useful in determining the cause of a woman's symptom.

(i) Frequency-volume chart (FVC): The recording of the time of each micturition and the volume voided for at least $24 \mathrm{~h}$. More useful clinical data are usually provided by 2 or 3 days of recording (not necessarily consecutive) $(3,5,29)$.

(ii) Bladder diary: The voiding diary is a 24-h record of the patient's fluid intake and output, pad usage, degree of incontinence, and episodes of incontinence $(3,29,38)$. Evaluation of a 3 - to 7 -day diary is also helpful clinically to plan the management (38).

The voiding diary identifies polyuria, defined as excessive urine production, which requires further investigation to exclude diabetes (23).

1. PVR: This volume is routinely measured during incontinence evaluation. After a woman voids, the PVR may be measured with a handheld sonographic scanner or by transurethral catheterization $(1,24,38)$. PVR test should be carried out within 10 min of a void, as it prevents the incidence of an artificially high result due to diuresis. A PVR volume of less than $50 \mathrm{~mL}$ is normal, and a volume of more than $200 \mathrm{~mL}$ is abnormal. Many women are unable to void well during an anxiety-ridden first visit. It is recommended to recheck the PVR volume at a future visit before embarking on further diagnostic tests $(23,31)$. If using a handheld scanner, care must be taken in women with an enlarged leiomyomatous uterus, as this may falsely record a large PVR. In these instances, or if a scanner is not available, transurethral catheterization may be used to confirm residual bladder volume $(24,31)$. A large PVR may often reflect one of several problems, including recurrent infection, urethral obstruction from a pelvic mass, or neurologic deficits. In contrast, a normally small PVR is often found in those with SUI (28). PVR is normal in SUI, UUI, and MUI.

2. Stress test/cough stress test/provocative stress test: It is an easy test that involves filling a patient's bladder to at least $300 \mathrm{~mL}$ or symptomatic fullness. This test is useful for diagnosing SUI, and considered positive when leakage is observed from the external urethral meatus during cough. The provocative stress test has demonstrated good sensitivity and specificity in diagnosing genuine SUI (GSUI) compared with other sophisticated testing methods (multichannel urodynamic studies). Scotti and Myers have reported that simple cystometry along with cough stress test is highly consistent in diagnosing GSUl. Wall et al. have reported that bladder filling without pressure measurement, combined with a cough stress test, is a simple, cost-effective, and consistent way of selecting patients with UUI related to detrusor overactivity from patients with straightforward SUI. Swift and Ostergard recommended that the cough stress remains a highly specific test, predominantly when combined with a prior negative cystometrogram $(5,39)$.

The supine empty stress test (SEST) is performed with the empty bladder and said to be positive if the patient loses urine on cough. Lobel and Sand found that the SEST had 65\%-70\% sensitivity and 67\%-76\% specificity for predicting ISD. The interpretation of cough stress test is negative if no leakages occur with the cough stress test. The cough stress test is considered positive if instantaneous leakage occurs with the cough stress test. The cough stress test is positive (mixed) if leakages occur in spurts, and detrusor overactivity is considered if delayed leakage occurs with flow. If leakage occurs with empty bladder in a supine position, ISD is regarded (39).

- Clinical use of stress test: A negative cough stress test rules out most cases of SUI. A positive cough stress indicates a good correlation with the presence of SUI. 
However, the diagnosis of SUI should be confirmed by multichannel studies if uninhibited detrusor contractions are observed during a cystometrogram, preceding the cough stress test.

Predominantly, if a conservative therapy is considered, the cystometrogram and cough stress test are not essential for a basic evaluation. However, in a more invasive treatment, the cystometrogram and cough stress test should be performed (39).

- Pad test: Incontinence can be confirmed by performing a pad weighing test.25 Pad testing is a noninvasive, simple, and effective method of quantifying the amount of urine loss in patients with UI. This test was initially proposed by Sutherst et al. in 1981 and modified by the Standardization Committee of the ICS in 1988. Pad testing may also be useful in documenting $U \mathrm{U}$ that is not discovered on clinical or urodynamic evaluation (40). Several pad tests have been described in the literature, such as short-term pad tests that last 15 min to $2 \mathrm{~h}$ and can be performed in the office and long-term pad tests that last 24-48 $\mathrm{h}$ and can be performed while patients go about their everyday life in their usual surroundings (41).

- Standardized 1-h pad test: The pad test is used for an objective quantification of UI. The subject is asked to drink $500 \mathrm{~mL}$ of water. Then, she applies a preweighed perineal pad to her perineum and spends the next hour walking around, performing normal household duties. She performs a series of exercises, including coughing and deep knee bending and washes her hands under running water before the pad is reweighed (3).

Versi et al. found that the 1-h pad test had a positive predictive value of $92 \%$ and a negative predictive value of $53 \%$ when screening for lower urinary tract dysfunction. Lose and Versi used the same test for diagnosing GSUI in patients who had no other urodynamic abnormality; the positive and negative predictive values were $91 \%$ and $72 \%$, respectively $(5,42)$.
2. Simple urodynamic tests (Level II): This level includes filling cystometry, voiding cystometry, and Q-tip test.

- Urodynamic studies (UDS): These tests are a dynamic assessment of the lower urinary tract and offer objective information about bladder and urethral function. However, they are invasive, embarrassing, expensive, and time-consuming. The aim of urodynamics is to achieve a diagnosis, and differentiating between SUI and OAB in a woman with a mixture of symptoms is arguably the most useful information these test provide (1).

- Indications for UDS: The indications for UDS are in patients with symptoms of voiding difficulty and neurological disease when conservative treatment has been tried and failed, prior to surgery, and when surgery has failed (1).

Despite these indications, UDS remain controversial. Leakage noted during testing is not always clinically relevant. In contrast, testing may be uninformative if the original offending maneuver or situation that led to incontinence cannot be reproduced during evaluation. Moreover, objective confirmation of the diagnosis is not always necessary, since empiric therapy in women with urge-predominant symptoms is reasonable (24).

- Simple cystometry: Objective measurements of bladder function are combined in a battery of tests termed cystometrics. Cystometrics may be simple or multichannel and differ in their sensitivity. Simple cystometrics allows determination of SUI and detrusor overactivity, as well as measurement of first sensation, desire to void, and bladder capacity. This procedure is easily performed using sterile water, a $60-\mathrm{mL}$ cathetertipped syringe, and urinary catheter, either Foley or Robnell. The urethra is sterilely prepared, the catheter is inserted, and the bladder is drained. A 60-mL syringe with its plunger removed is attached to the catheter and filled with sterile water. Water is added in increments until a woman feels: (1) a sensation of bladder filling, (2) an urge to void, and (3) her bladder maximum capacity. 
Water volumes are noted at each three of these points. Changes in the fluid meniscus within the syringe are monitored. Any meniscus elevation indicates bladder contraction and establishes a diagnosis of detrusor overactivity. Once bladder capacity is reached, the catheter is removed, and the woman is asked to perform a Valsalva maneuver or cough while standing. Leakage indicates SUI. Advantageously, simple cystometrics are easy to perform, require inexpensive equipment, and can typically be completed by most gynecologists. The limitation of simple cystometric testing, however, is its inability to reflect changes found with ISD (24).

Simple cystometry is subject to two main sources of error. First, the intravesical pressure cannotbe measured constantly during bladder filling. So, sequential bladder filling must be employed. Second, measurement of the intravesical pressure does not always precisely represent changes in detrusor pressure. Hence, subtracted cystometry is required, which involves measuring the intravesical and the intraabdominal pressure simultaneously (3). The information that is obtained from a subtracted cystometrogram includes sensation, capacity, contractility, and compliance (25). The values for cystometry are as follows: normal: residual urine $<50 \mathrm{~mL}$; first desire to void: $150-250 \mathrm{~mL}$; cystometric capacity: 400-600 mL; maximum detrusor pressure: filling: $<15 \mathrm{~cm}$ water (voiding: $<70 \mathrm{~cm}$ of water; multichannel: only $10 \%$ patients require it); peak flow rate: $>15 \mathrm{~mL} / \mathrm{s}$; single channel (sensitivity 100\%; specificity 51\%) and multichannel: only 10\% patients require it $(3,38)$.

-Voiding cystometry: The filling catheter is removed before voiding to prevent any unnecessary urethral obstruction following the completion of filling cystometry. Allowing simultaneous measurement of detrusor pressure along with the urine flow rate, the intravesical and rectal pressure recording lines are left in situ. As with uroflowmetry, the patient is asked to void while sitting on a flowmeter in private. During normal voiding, a coordinated contraction of the bladder and at the same time relaxation of the urethra are observed, which are sustained until the bladder is empty. Women normally void with a detrusor pressure of less than $60 \mathrm{~cm} \mathrm{H} 20$ and a peak flow of $>15$ $\mathrm{mL} / \mathrm{s}$ for a voided volume of at least $150 \mathrm{~mL}$. If detrusor pressure during voiding is reduced with low flow rates and a noteworthy postmicturition residual is observed then the patient is considered to have voiding difficulty. In women, voiding problems are probably secondary to impaired detrusor contractility and infrequently because of bladder outflow obstruction. Bladder outflow obstruction is exemplified by a raised detrusor pressure during voiding and low flow rate. Furthermore, the patient may use additional abdominal straining to improve the intravesical pressure.

Detrusor sphincter dyssynergia is pathological contraction of the external sphincter that occurs during a bladder contraction, mainly in women with overt neurological disease. Typically, a high detrusor pressure is observed during voiding related to poor flow rate. In a few women, urinary retention may happen, and therefore catheterization is necessary (3). The results of urodynamic investigation may be confounded by discomfiture or an unknown testing environment, as both relaxation of the urethral sphincter and initiation of voiding are subject to cortical influence (3).

Subtracted cystometry is performed to know true detrusor pressure (DP). The true DP is equal to intravesical-intraabdominal pressure. The normal value is $\leq 10 \mathrm{~cm} \mathrm{H2O}$ (300 mL) and $\leq 15 \mathrm{~cm} \mathrm{H20} \mathrm{(500} \mathrm{mL),} \mathrm{with} \mathrm{no} \mathrm{abnormal}$ detrusor activity during filling (38).

- Q-Tip test: This test was introduced by Crystle et al. (1972). It measures urethrovesical junction (UVJ) mobility (43). If a urethra is poorly supported, it may display hypermobility during increases in intra-abdominal pressures. To assess mobility, a clinician places the soft end of a cotton swab into the urethra to the UVJ. Failure to insert the swab to this depth typically leads to errors in the assessment of the UVJ support. Termed the Q-tip test, this evaluation may be uncomfortable, and application of intraurethral analgesia may prove helpful. Commonly, 1\% lidocaine jelly 
is placed on the cotton swab prior to insertion. Following placement, Valsalva maneuver is prompted, and the swab angle excursion at rest and with Valsalva maneuver is measured using a goniometer or standard protractor. An angle excursion at rest or with Valsalva maneuver greater than 30 degrees above the horizontal indicates urethral hypermobility, and may help in the direct planning of surgical treatment for SUI $(5,24,43)$.

- Bonney's test: This test aims to see whether an uplift of the urethrovesical function stops the incontinence during times of raised intra-abdominal pressure, thus guiding the type of surgery required. With the patient in a dorsal position, light pressure is applied on the sides of the upper urethra without causing an uplift. A positive test is indicated by the control of urinary leakage from the vagina with closure of the internal sphincter by pressure. The usefulness of this test is not to demonstrate the patient remaining dry but to find the patient who continues to leak in spite of the correction of normal anatomic support and is thus suspicious of having a significant element of intrinsic urethral sphincter weakness of deficiency (44).

\section{Complex urodynamic test (Level III): Certain patients require} complex testing before surgical therapy can be performed. Patients who may benefit from multichannel subtracted urodynamic testing are older than 50 years of age with a history of radical pelvic surgery, pelvic irradiation, or both, incontinence or prolapse surgery, severe POP, no evidence of anterior vaginal relaxation, continuous incontinence, no urethral hyper mobility, hematuria with negative urine culture, possible urethral diverticulum, and inconclusive or confusing office evaluation (5).

Complex urodynamic test includes multichannel cystometry, pressure flow voiding studies, urethrocystoscopy, and urethral pressure profile.

- Multichannel cystometrics: As noted, simple cystometric testing does not identify ISD. This determination is important, since this diagnosis may potentially preclude certain surgical options. Additionally, multichannel cystometrics provides more information on other physiologic parameters of the bladder, not afforded by simple cystometrics. Multichannel cystometrics is more commonly performed by urogynecologists or urologists due to limited equipment availability and increased costs. Testing can be performed with a woman standing or seated upright in a specialized urodynamics evaluation chair $(7,24)$. During testing, two catheters are used. One is placed into the bladder and the other into either the vagina or the rectum. The vagina is preferred unless advanced prolapse is evident because stool in the rectal vault may obstruct catheter sensors and lead to inaccurate readings. Additionally, vaginal placement for most women is more comfortable (24). Using catheters with a special transducer, the patient's bladder is filled with sterile saline at $75-100 \mathrm{~mL} / \mathrm{min}$. As the bladder is filled, the patient is instructed to note when she senses the first urge to void, a full bladder, and maximum holding capacity. Bladder and true DPs are measured during filling, and detrusor activity is recorded. True DP is calculated by subtracting abdominal pressure from bladder or vesical pressure. An increase in true DP associated with leaking and urgency confirms detrusor instability. Provocative testing is usually performed if bladder instability has not yet been demonstrated. Once a full bladder capacity is achieved or $300 \mathrm{~mL}$ has been infused, filling is stopped and the patient is asked to cough and then perform Valsalva maneuver. If these fail to stimulate a detrusor contraction, further provocative testing with running water can be performed. If SUI or detrusor instability has not been documented at this point, the patient should be challenged in the standing position. With a full bladder, the standing patient is asked to cough, bear down, heel bounce, and listen to the running water. Bladder stability is then confirmed if no detrusor contractions are documented in the sitting or standing position (5,24).

Ambulatory cystourethrovaginometry is an alternative to stationary cystometry. Ambulatory testing was developed to enhance detection of patients who have incontinence and who are unable to demonstrate their 
leakage in the urodynamics lab. The ambulatory setup and catheter placement are similar to those used for stationary cystometry. The catheters, however, must be either taped or sutured in place. Leakage can be recorded using pads with special sensors or conductance rings on the catheter. Advantages of ambulatory cystometry also include antegrade bladder filling, which is more natural. Patients may also feel more relaxed about demonstrating incontinence episodes if the stress of performing is removed (5).

- Uroflowmetry: Initially, women are requested to empty their bladder into a commode connected to a flowmeter (uroflowmetry). After a maximal flow rate is recorded, the patient is catheterized to measure a PVR as well as to ensure an empty bladder prior to further testing. This test provides information on a woman's ability to empty her bladder. It can identify women with urinary retention and other types of voiding dysfunction (24). In women, for a volume of $150 \mathrm{~mL}$ of urine voided, the normal recording is a bell-shaped curve with a peak flow rate of at least $15 \mathrm{~mL} / \mathrm{s}$. In an asymptomatic woman undergoing incontinence surgery, a reduced flow rate may be important, as she is more likely to develop voiding difficulties in the postoperative period $(3,5)$.

- Cystometrography: Following uroflowmetry, cystometrography is performed to determine whether a woman has urodynamic evidence of SUI or detrusor overactivity (DO). Moreover, this test provides information on bladder threshold volumes at which a woman senses bladder capacity. Delayed sensation or a sensation of bladder fullness only with large capacities may indicate neuropathy $(5,24)$. Conversely, extreme bladder sensitivity may suggest sensory disorders such as IC. For the cystometrogram, a 6-F microtransducer catheter is inserted transurethrally into the bladder, and a second catheter is inserted into the vagina. For women with advanced prolapse who may not be able to accommodate or retain the second catheter in their vagina, the second catheter is placed in the rectum.
The bladder is filled with $0.9 \%$ NS at a rate of $100 \mathrm{~mL} /$ min using a cystometric pump. During filling, a woman is asked to cough at each $50-\mathrm{mL}$ interval. Additionally during filling, the volumes at which the first desire to void and maximal bladder capacity are reported are recorded (24).

From pressure readings, DO and/or urodynamic SUI may be identified. Once $200 \mathrm{~mL}$ of saline has been instilled, an abdominal leak point pressure is measured. The patient is asked to perform a Valsalva maneuver, the pressure generated by the effort is measured, and evidence of urine leakage is sought. If leakage is seen when a pressure of less than $60 \mathrm{~cm} \mathrm{H2O}$ is generated, then criteria have been met for a diagnosis of intrinsic sphincteric deficiency. Using the true zero of intravesical pressure as the baseline, at a bladder volume of $200 \mathrm{~mL}$, abdominal leak point pressures are measured. However, the volume at which this test is performed varies from institution to institution, with some choosing to use bladder capacity and others choosing to use $150 \mathrm{~mL}$ as the testing volume. Readings are obtained or calculated and include: (1) intra-abdominal pressure, (2) vesicular pressure, (3) calculated DP, (4) bladder volume, and (5) saline infusion flow rate. From these catheter readings, information regarding bladder, intra-abdominal, and DPs can be obtained $(5,24)$.

- Pressure flowmetry: This evaluation usually follows cystometrography and is similar to the uroflowmetry conducted at the beginning of urodynamic testing. A woman is asked to void into a beaker that rests on a calibrated weighted sensor. Maximum flow rate and PVR are once again recorded. However, during voiding, a woman now has a microtip transducer catheter in her bladder, which provides additional information regarding DP at maximum flow. This is particularly useful in women who may have incomplete bladder emptying. As noted earlier, in women with urinary retention, the offending source may be obstruction or poor detrusor contractility $(3,5,24)$. 
- Urethral pressure profilometry: The final part of UDS testing is the urethral pressure profile (UPP) (24). The resting UPP is a graphical record of pressure within the urethra at successive points along its length (3). Usually in this test, $200 \mathrm{~mL}$ volume is instilled in the bladder. However, again, this volume is often institution dependent. This test provides valuable information on the intrinsic properties of the urethra and aids in diagnosing ISD. A diagnosis of ISD is made if the MUCP is $20 \mathrm{~cm} \mathrm{H2O} \mathrm{or} \mathrm{less} \mathrm{(24).}$

- Stress leak point pressure: This involves a dynamic assessment of urethral function in a patient with GSUI. With $150 \mathrm{~mL}$ of fluid in the bladder, the patient is asked to gradually bear down. The Valsalva pressure at which leakage occurs is measured. If leakage occurs at a relatively low pressure, that is, less than $60 \mathrm{~cm} \mathrm{H2O}$, intrinsic urethral function is poor, and a sling procedure should be considered $(7,23)$.

- Urethrocystoscopy: Endoscopic evaluation of the urethra and bladder can detect urethral inflammation, urethral diverticulum, $I C$, and various bladder lesions. This procedure can be performed easily in the office setting if appropriate training for the examiner has been completed. If a urethral diverticulum is suspected or a postoperative patient presents with postoperative symptoms that cause concern, cystoscopy should be performed. However, it has limited use in diagnosing GSUI (5).

- Video cystourethrography: It is the single most informative investigation that combines cystometry, uroflowmetry, and radiological screening of the bladder and urethra (25). The whole investigation can be recorded on a video tape or computer with a sound commentary for immediate and later replay, which helps in facilitating diagnosis, audit, data storage, research, and education. It is particularly useful for patients in whom previous incontinence surgery has failed or who have mixed or unusual symptoms and neurological disorders. However, in other conditions, it has no advantage over subtracted cystometry (3).

\section{CONCLUSION}

UI can be a physically and mentally devastating disorder. SUI, UUI, and MUI are the three most common types, and of these, SUl is the most common in women. Obstetrician-gynecologists play an imperative role in diagnosing and treating SUI. The American Urogynecologic Society recommends history taking, urine examination, physical assessment along with POP, cough stress test, evaluation of urethral mobility, and measurement of PVR urine volume for assessing a patient with symptoms of uncomplicated SUI. However, in women with complicated SUI additional diagnostic evaluation with multichannel urodynamic testing mainly before surgical treatment is beneficial. The health care provider's decision regarding clinical judgment must direct to refer the patient to an expert with appropriate training and skills in female pelvic medicine and reconstructive surgery or to perform preoperative multichannel urodynamic testing.

\section{REFERENCES}

1. Magowan BA, Owen P, Drife J. Clinical Obstetrics and Gynecology. 2nd ed. New York: Saunders Elsevier; p 175-80, 2009.

2. Bump RC, Norton PA. Epidemiology and natural history of pelvic floor dysfunction. Obstetrics and gynecology clinics of North America 1998; 25:723-46.

3. Edmonds DK., the editor. Dewhurst's Textbook of Obstetrics and Gynaecology. USA: Blackwell publishing; 2007.p. 504-518.

4. DeLancey JOL. The pathophysiology of stress urinary incontinence in women and its implications for surgical treatment. World J Urol 1997;15:268-274.

5. Ramson SB. Practical Strategies in Obstetrics and Gynecology. Philadelphia: W B Saunders; p 150, 2000.

6. Chene G, Mansoor A, Jacquetin B, Mellier G, Douvier S, Sergent $F$, Aubard Yves, Seffert P. Female urinary incontinence and intravaginal electrical stimulation: an observational prospective study. European Journal of Obstetrics and Gynecology and Reproductive Biology 2013;170:275-80.

7. Vasavada SP, Carmel ME, Rackley R. Urinary incontinence. Updated: Jan 28, 2013(cited 2013Feb17). Available from: http://emedicine.medscape.com/article/452289overview\#aw2aab6b2b1aa.

8. Herbruck LF. Urinary Incontinence in the Childbearing Woman. UrolNurs 2008; 28(3):163-171.

9. Brunenberg $D E$, Joore $M A$, Veraart $C P$, Berghmans $B C$, van der Vaart $\mathrm{CH}$, Severens JL. Economic evaluation of duloxetine for the treatment of women with stress urinary incontinence: a Markov model comparing pharmacotherapy with pelvic floor muscle training. Clin Ther. 2006;28(4):604-18.

10. Ivan F, Štefica FG, Nikola F. Minimally invasive laser procedure for early stages of stress urinary incontinence (SUI). J LAHA; 2012;1:67-74. 
11. Athanasopoulos A, Perimenis P. Pharmacotherapy of urinary incontinence. Int Urogynecol J Pelvic Floor Dysfunct 2009; 20:475-82.

12. Farrell SA, Epp A, Flood C, Lajoie F, MacMillan B, Mainprize $T$, Robert $M$. The evaluation of stress incontinence prior to primary surgery. J Obstet Gynecol Can 2003;25:313-8.

13. Hong $\mathrm{JH}$, Choo $\mathrm{M}$, Lee $\mathrm{K}$. Long-term results of Laparoscopic Burch Colposuspension for Stress Urinary Incontinence in Women.Korean Med Sci 2009; 24:1182-1186.

14. Dwyer NI. Stress urinary incontinence in women. Hospital physician board review manual. Urology 2006;13:1-12.

15. Bakar Y, Cinar OO, Ozengin N, Duran B. The use of extracorporeal magnetic innervation for the treatmentof stress urinary incontinence in older women: a pilot study. Arch Gynecol Obstet 2011;284:1163-1168.

16. Harvey MA, Kristjansson B, Griffith D, Versi E. The Incontinence Impact Questionnaire and the Urogenital Distress Inventory: A revisit of their validity in women without an urodynamic diagnosis. Am J Obstet Gynecol 2001;185:25-31.

17. Price DM, Noblett K. Comparison ofthe cough stress test and 24-h pad test inthe assessment of stress urinaryincontinence. IntUrogynecol J 2012;23(4):429-33.

18. Serati M, Uccella S,SalvatoreS.Stress urinary incontinence in women of childbearing age. European Urological Review 2008; 72-4.

19. Dumoulin C, Bourbonnais D, Morin M, Gravel D, Lemieux M. Predictors of Success for Physiotherapy Treatment in Women With Persistent Postpartum Stress Urinary Incontinence.Arch Phys Med Rehabil 2010;91:1059-63.

20. Kerr LA. Bulking agents in the treatment of stress urinary incontinence: history, outcomes, patient populations, and reimbursement profile. Rev Urol 2005; 7: S3-S11.

21. Richter HE, Litman HJ, Lukacz ES, Sirls LT, Rickey L, Norton P, et al. Demographic and clinical predictors of treatment failure one year after midurethral sling surgery. Urinary Incontinence Treatment Network. Obstet Gynecol 2011;117:913-21.

22. Evaluation of uncomplicated stress urinary incontinence in women before surgical treatment. Committee Opinion No. 603. The American College of Obstetricians and Gynecologists. Obstet Gynecol 2014;123:1403-7.

23. Mishell DR, Goodwin TM, Brenner PF. Management of Common Problems in Obstetrics and Gynecology. USA: Blackwell publishing; pp 287-9, 290, 2002.

24. O'Schorge J, Schaffer JI, Halvorson LM, Hoffman BL, Bradshaw KD, Cunningham FG. William's Gynecology. New York: McGraw Hill; p 512-22, 2008.

25. Paick JS, Kim SW, Oh SJ, Ku JH. A generic health-related quality of life instrument, the Medical Outcomes Study Short Form36 , in women with urinary incontinence. Eur J Obstet Gynecol Reprod Biol 2007;130:18-24.

26. Urinary incontinence in women. ACOG Practice Bulletin No. 63. American College of Obstetricians and Gynecolo-gists. Obstet Gynecol 2005;105:1533-45.

27. Tannenbaum C, Brouillette J, Corcos J. rating improvements in urinary incontinence: do patients and their physicians agree? Age and Ageing 2008;37:379-383.
28. Fultz NH, Burgio K, Diokno AC, Kinchen KS, Obenchain R,Bump RC. Burden of stress urinary incontinence for communitydwelling women. Am J Obstet Gynecol 2003;189:1275-82.

29. Haylen $B T$, de Ridder $D$, Freeman RM, et al. An International Urogynecological Association (IUGA)/International Continence Society (ICS) joint report on the terminology for female pelvic floor dysfunction. Neurourol Urodyn 2010;29:4-20.

30. Abrams P, Blaivas JG, Stanton SL, et al. The standardisation of terminology of lower urinary tract function. Scand J Urol Nephrol Suppl 1988;114:5-19.

31. Nygaard IE. Stress Urinary Incontinence. Obstet Gynecol 2004;104:607-20.

32. Yang A, Mostwin J, Genadry R, et al. Patterns of prolapse demonstrated with dynamic fastscan MRI; reassessment of conventional concepts of pelvic floor weaknesses. Neurourol Urodyn 1993;12:310-1.

33. Victoria L, Handa G, Garrett E, Hendrix S, Gold E, Robbins J. Progression and Regression of pelvic organ prolapse: $A$ longitudinal study of menopausal women. Am J Obstet Gynecol 2004; 190: 27-32.

34. Richard C, Bump, Mattiasson A, Kari B, Linda P, Brubaker M et al. The standardization of terminology of female pelvic organ prolapse and pelvic floor dysfunction. Am J Obstet Gynecol 1996; 175:10-17.

35. Mouritsen L. Classification and evaluation of prolapse. Best practice and Research Clinical Obstetrics and Gynaecology 2005; 19: 895-911.

36. Messelink B, Benson T, Berghmans B, et al. Standardization of terminology of pelvic floor muscle function and dysfunction: Report from the Pelvic Floor Clinical Assessment Group of the International Continence Society. Neurourol Urodyn 2005;24:374-80.

37. Dietz HP, Shek KL. Validity and reproducibility of the digital detection of levator trauma. Int Urogynecol J 2008;19:1097101.

38. Arora R, Goswami D, Batra S. Practical approach to management of common gynecological diseases. New Delhi: Jaypee Brothers; pp 106-8, 2008.

39. Ghoniem G, Stanford E, Kenton K, Achtari C, Goldberg R, Mascarenhas $T$, et al. Evaluation and outcome measures in the treatment of female urinary stress incontinence: International Urogynecological Association (IUGA) guidelines for research and clinical practice. Int Urogynecol J Pelvic Floor Dysfunct 2008; 19: 5-33.

40. Wu W, Sheu B, Lin H. Twenty-minute pad test: Comparison of infusion of $250 \mathrm{ml}$ of water with strong-desire amount in the bladder in women with stress urinary incontinence European Journal of Obstetrics and Gynecology and Reproductive Biology 2008;136:121-125.

41. Ryhammer AM, Djurhuus JC, Lauberg S. Pad testing in incontinence women: a review. Int Urogynecol J 1999;10:111-5.

42. Lose G, Versi E. Pad-weighing tests in the diagnosis and quantification of incontinence. Int Urogyneeol J 1992;3:324-32.

43. Smith GK. The history of spina bifida, hydrocephalus, paraplegia, and incontinence. Pediatr Surg Int 2001;17:424-32.

44. Saraiya UB, Rao KA, Chatterjee A, the ed. Principles and Practice of Obstetrics and Gynecology for postgraduates. New Delhi: Jaypee Brothers; p 360, 2003. 